\title{
Die Schweizerische Kopfwehgesellschaft SKG
}

\section{Peter Sandor}

PD Dr. med., Präsident der SKG, Oberarzt, Leiter Abt. Kopfweh und Schmerz, Neurologische Klinik, UniversitätsSpital Zürich

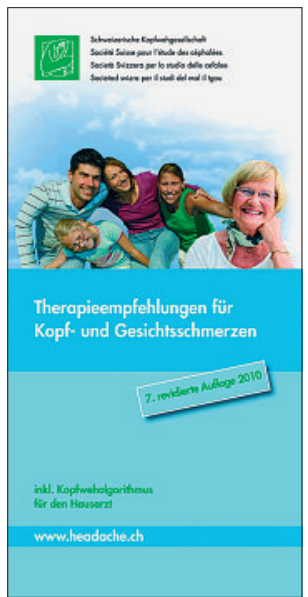

Korrespondenz:

Administrative Geschäftsstelle der SKG

Gabriela Deutsch c/o IMK Institut für Medizin und Kommunikation AG

Münsterberg 1

CH-4001 Basel

Tel. 0612713551

Fax 0612713338

kopfweh@imk.ch
Die Schweizerische Kopfwehgesellschaft SKG wurde 1995 gegründet und vereint Spezialärzte, Grundversorger und Wissenschafter, die sich mit dem Thema Kopfschmerzen befassen. Sie verfolgt das Ziel, die Forschung, Diagnostik und Therapie von Kopfschmerzen zu fördern und den neuesten Wissensstand an Ärzte, Patienten und Wissenschafter weiterzugeben. Zudem ist die administrative Geschäftsstelle der Gesellschaft oft erste Ansprechpartnerin für Patienten und Betroffene. Die SKG ist Mitglied der «European Headache Federation» und gehört seit 1996 der «International Headache Society» an.

Gemeinsam mit ihren rund 150 Mitgliedern, überwiegend Neurologen, aber auch Internisten und Psychiater, verfolgt die SKG folgende Ziele:

- Förderung der wissenschaftlichen Arbeit und Forschung im Bereich der Kopfschmerzen

- Verbesserung der Zusammenarbeit von Ärzten und Angehörigen von Berufsgruppen, die in der Behandlung und Betreuung von Kopfwehpatienten engagiert sind oder Kopfwehforschung betreiben

- Information von Betroffenen und ihrer Angehörigen über neuste Therapiemöglichkeiten

Somit bietet die SKG nicht nur Kopfwehspezialisten und Grundversorgern, sondern auch Patienten eine vielseitige Plattform zum Umgang mit den Kopfschmerzen und deren Behandlung an.

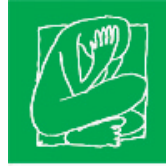

Schweizerische Kopfwehgesellschaft Société Suisse pour l'étude des céphalées Società Svizzera per lo studio delle cefalee Societad svizra per il studi del mal il tgau

\section{Kongresse}

Bei den jährlichen Kongressen arbeitet die SKG eng mit anderen Gesellschaften zusammen, um die interdisziplinäre, kollegiale Kommunikation und den Wissensaustausch zu fördern und Synergien auszubauen.

Die SKG wird anlässlich des 1. Kongresses der Swiss Federation of Clinical Neuro-Societies SFCNS vom 2. bis 4. Juni 2010 in Basel eigens einen Nachmittag zum Thema Kopfschmerzen gestalten.

Am 18. November 2010 treffen sich die Mitglieder zur 26. Jahrestagung in Zürich und der Kongress 2011 wird in Kooperation mit der Schweizerischen Gesellschaft für Neurochirurgie organisiert.

\section{Webseite}

Die dreisprachig verfasste SKG-Webseite richtet sich gleichermassen an Fachpersonen wie auch an Patienten. In einer eigenen Rubrik für Betroffene werden unter anderem über 30 Fallbeispiele von Kopfwehpatienten geschildert und mit den entsprechenden Therapiemöglichkeiten untermauert. Auch Ärzten und Therapeuten, die Kopfwehpatienten betreuen, dient

\section{Die SKG arbeitet eng mit anderen Gesellschaften zusammen, um die interdisziplinäre, kollegiale Kommunikation und den Wissens- austausch zu fördern und Synergien auszubauen}

\section{Therapiekommission}

Die Therapiekommission setzt sich aus Fachpersonen zusammen, die über eine besondere Expertise bei der Behandlung von Kopfwehpatienten verfügen. Eine ihrer Aufgaben ist die regelmässige Herausgabe von Empfehlungen über die gebräuchlichen und neusten Kopfschmerztherapien. Die nächste Neuauflage erscheint im Frühling 2010 und befasst sich neu mit Themen wie Kinderkopfschmerzen, chirurgische Eingriffe bei Kopfschmerzen und umstrittene Verfahren.

Des Weiteren sind Richtlinien zur Beurteilung der Arbeitsunfähigkeit infolge Kopfschmerzen in Vorbereitung. die Webseite als Unterstützung in der täglichen Arbeit. Sie enthält Empfehlungen für die tägliche Praxis und Informationen über neue Trends und Möglichkeiten, teils mit kritischer Diskussion seitens unserer Spezialisten. Ausserdem sind Dokumente wie beispielsweise der Kopfwehkalender frei für den Download verfügbar.

Weitere Informationen zur Schweizerischen Kopfwehgesellschaft SKG erhalten Sie auf der Webseite unter www.headache.ch oder bei der administrativen Geschäftsstelle. 